\title{
LETTERS
}

\section{Increased glutaredoxin-1 and decreased protein S-glutathionylation in sputum of asthmatics}

\section{To the Editor:}

Total reduced glutathione (GSH), the main pulmonary antioxidant, is increased in asthma patients and, in some studies, increased amounts of oxidised glutathione (GSSG) were also found. In addition, enzymes that regulate the GSH redox cycle are altered in asthmatics (reviewed in [1]).

GSH, with its redox cycle partners, serves to maintain the reduced state of protein thiols, which can be achieved by scavenging oxidants or by the covalent reversible binding of GSH to protein thiols. The latter occurs under physiological conditions, is induced upon mild oxidative stress and is known as S-glutathionylation (PSSG) [2]. PSSG protects targeted thiols from irreversible oxidations and can modulate protein function. Of significant relevance in asthma, SERCA (sarco/ endoplasmic reticulum calcium ATPase) is activated by PSSG, increasing smooth muscle relaxation, and PSSG of the RyR (ryanodine receptor) calcium channel was associated with impaired coupling. With respect to inflammation, nuclear factor- $\kappa \mathrm{B}$ and activator protein-1 are negatively affected by PSSG (reviewed in [2]).

GSH can be removed from proteins by glutaredoxins (Grx), which restores the function of proteins targeted by PSSG [2]. The Grx1 isoform localises to the cytosol, and Grx2 to mitochondria and the nucleus. Grx1 can also reduce low molecular weight disulfides and, thus, proteins with functionally important disulfide bonds could also be affected by Grx1 alterations.

In contrast to damaging oxidations and measurements of GSH, PSSG and Grx have rarely been studied in lung diseases, and never in conjunction. In chronic obstructive pulmonary disease (COPD), Grx1-positive alveolar macrophages were negatively correlated with forced expiratory volume in $1 \mathrm{~s}$ (FEV1) and sputum Grx1 levels were higher during exacerbations [3]. In a murine model of allergic airway disease, we found increased Grx1 expression and activity [4].

Given the importance of oxidative stress in the pathogenesis of asthma and the critical role GSH homeostasis plays therein, we conducted a study evaluating Grx1 and PSSG levels in induced sputum from 33 asthmatics and nine healthy controls (table 1).

Sputum was induced by inhalation of hypertonic saline and Grx1 in the supernatant was analysed by Western blotting. To determine PSSG content, 1\% sodium borohydride was used to remove GSH from sputum supernatant protein pellets and GSH was quantified using the Ellman's reagent GSSG reductase recycling method.

RNA was extracted from ciliated epithelial cells isolated during bronchoscopy in three healthy controls and three asthma patients. Quantitative PCR was performed using primers for Grx1, Grx2 and glutaraldehyde phosphate dehydrogenase. Relative quantity was calculated using the comparative threshold cycle method.

The level of Grx1 protein in sputum was significantly increased in asthmatics compared with controls (fig. 1). Interestingly, an extra band below Grx1 was observed in 32\% of patients. Grx2 was undetectable. Consistent with the function of Grx1, the PSSG level was significantly decreased in sputum of asthmatics and negatively correlated with Grx1 ( $\mathrm{r}=0.518, \mathrm{p}=0.001)$.

We next categorised asthmatics as eosinophilic, neutrophilic, paucigranulocytic or mixed based on published values for sputum eosinophil percentages [5] and the internal reference value of $76 \%$ for neutrophils established in our clinic, since an intricate relationship between Grx/PSSG and inflammation has been observed. Thus, 15 patients were labelled eosinophilic, eight neutrophilic, 10 paucigranulocytic and none as mixed. Lung function, asthma control and quality of life did not differ between cellular phenotypes. Grx1 protein levels were specifically enhanced in eosinophilic and paucigranulocytic but not in neutrophilic asthmatics. PSSG levels were decreased only in eosinophilic and neutrophilic asthmatics compared with healthy controls. We then assessed associations with total and differential cell percentages in induced sputum. Total cell numbers were found to be positively related to Grx1 levels $(r=0.425, p=0.006)$ and negatively to PSSG $(r=-0.632$, $\mathrm{p}<0.001)$. PSSG levels were positively related to epithelial cell $(\mathrm{r}=0.601, \mathrm{p}<0.001)$, squamous cell $(\mathrm{r}=0.355, \mathrm{p}=0.025)$ and lymphocyte percentages $(r=0.362, p=0.022)$, and negatively related to the percentage of viable cells $(r=-0.441, p=0.004)$ in the entire study population and in asthma patients. Grx1 sputum levels did not correlate with specific sputum cell types. Since we demonstrated increased Grx1 expression in airway epithelial cells in a mouse model of allergic airway disease, we investigated Grx mRNA expression in primary bronchial epithelial cells obtained from asthmatics and controls. A higher level of Grx1 mRNA expression was indeed present in epithelial cells isolated from asthmatics compared with cells isolated from controls. Grx2 mRNA levels were not different.

The increased Grx1 protein levels in the induced sputum of asthmatics could be part of the protective response of the lungs to oxidative stress. Enhancing extracellular Grx1 could serve to increase free GSH directly as opposed to via transcriptional upregulation of $\gamma$-glutamylcysteinyl ligase. The protein thiols could be returned to their reduced state, which is unlikely since the GSH/GSSG ratio is not increased in asthma [1]. Alternatively, loss of this protective modification would leave proteins susceptible to damaging oxidations. Carbonylated 
TABLE 1 Demographic and functional characteristics

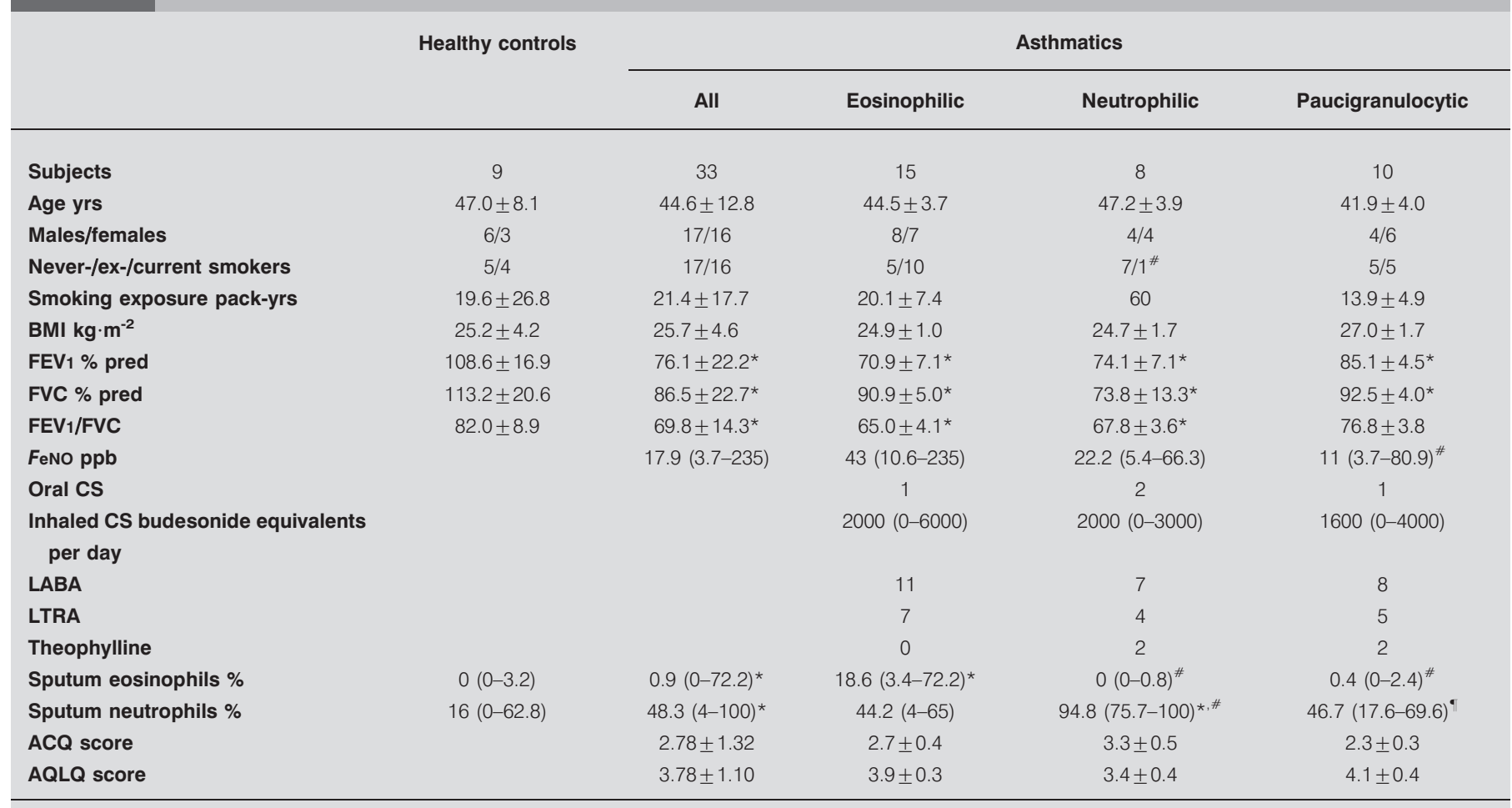

Data are presented as $\mathrm{n}$, mean \pm SD or median (range). BMI: body mass index; FEV1: forced expiratory volume in $1 \mathrm{~s} ; \%$ pred: \% predicted; FVC: forced vital capacity; FeNO: exhaled nitric oxide fraction; CS: corticosteroids; LABA: long-acting $\beta$-agonists; LTRA: leukotriene receptor antagonist; ACQ: Asthma Control Questionnaire; AQLQ: Asthma Quality of Life Questionnaire. *: $p<0.05$ versus healthy control subjects; ${ }^{*}: p<0.05$ versus eosinophilic asthma; ${ }^{\circ}: p<0.05$ versus neutrophilic asthma.

albumin and $\alpha_{1}$-antitrypsin have indeed been detected in sputum of asthmatics [6].

In human lungs, Grx1 has been demonstrated predominantly in macrophages and epithelial cells [7]. Here, Grx1 levels did not correlate with the macrophage percentage in induced sputum, but a higher level of Grx1 mRNA expression was found in primary bronchial epithelial cells from asthmatics compared with controls, making bronchial epithelial cells the most likely source of sputum Grx1. Furthermore, in vitro studies have demonstrated Grx1 in culture supernatants, which reflected expression profiles [8], and probably involves active nonclassical secretion.

In this study, we also show that sputum PSSG levels were significantly decreased in patients with asthma, especially in the two inflammatory phenotypes. However, no correlation with the percentage of eosinophils or neutrophils was observed. This is in agreement with an earlier observed lack of PSSG reactivity in neutrophils [9]. The likelihood of epithelial cells as a source of Grx1 in sputum contrasts with the positive relationship of sputum epithelial cells to PSSG, although epithelial cells in sputum could represent a different pool from those isolated by brushing. These data indicate that PSSG is not only influenced by Grx1 levels but probably also oxidative stress related to the inflammatory state of the lungs. Anti-inflammatory therapy did not relate to PSSG or Grx1 levels.
Importantly, FEV1 \% predicted negatively correlated with sputum Grx1 levels $(\mathrm{r}=-0.314, \mathrm{p}=0.04)$ and positively with PSSG $(r=0.336, p=0.032)$ in the whole study group. When restricting the analyses to all asthma patients, these correlations did not remain significant. However, Grx1 in eosinophilic asthmatics specifically still negatively correlated with FEV1 \% pred $(\mathrm{r}=-0.532, \mathrm{p}=0.04)$ and PSSG correlated positively with FEV1 \% pred in neutrophilic patients $(r=0.750, p=0.05)$.

Thus, a better lung function is associated with lower Grx1 and higher PSSG levels in induced sputum. Conversely, in COPD, Grx1-positive macrophages in lung tissue positively correlated with FEV1, while there was no information on PSSG [3]. Here, we lack information on PSSG in specific cell types and it is difficult to extrapolate these findings to the Grx/PSSG axis in lung tissue.

In addition to its positive correlation with FEV1 in neutrophilic asthmatics, PSSG also negatively correlated to the degree of disease control in this phenotype as assessed by the Asthma Control Questionnaire $(r=-0.750, p=0.05)$. Therefore, sputum PSSG is linked to neutrophilic asthma and associated with disease control.

In addition to studying alterations in mRNA and protein levels, classical post-translational modifications and generic antioxidants, these data show that PSSG of targets relevant to asthma should be further investigated as they could play a key role in pathophysiology and, possibly, treatment, since 
a)

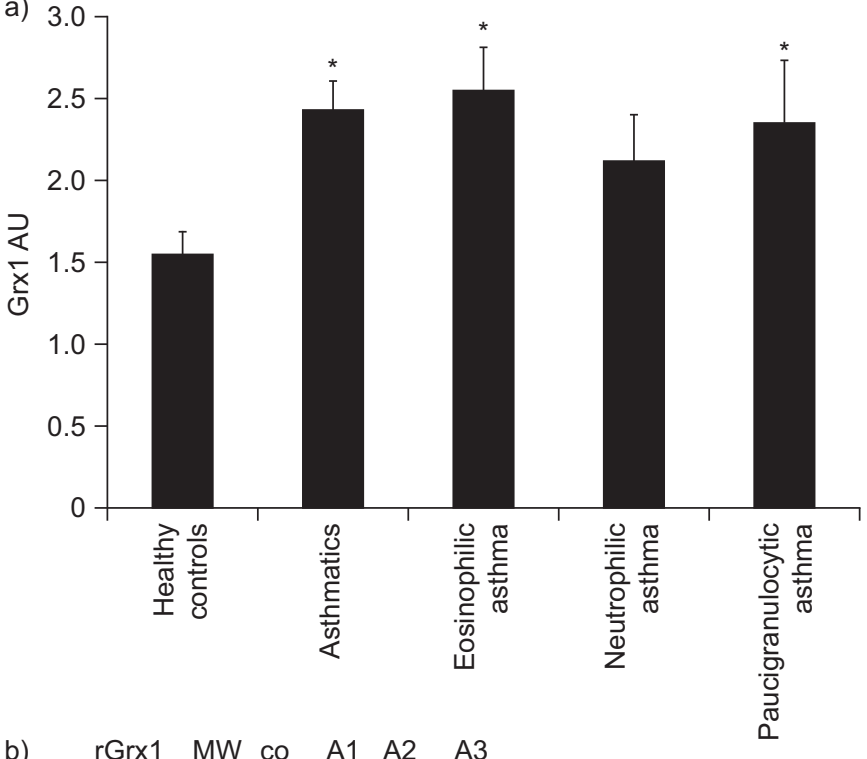

b)
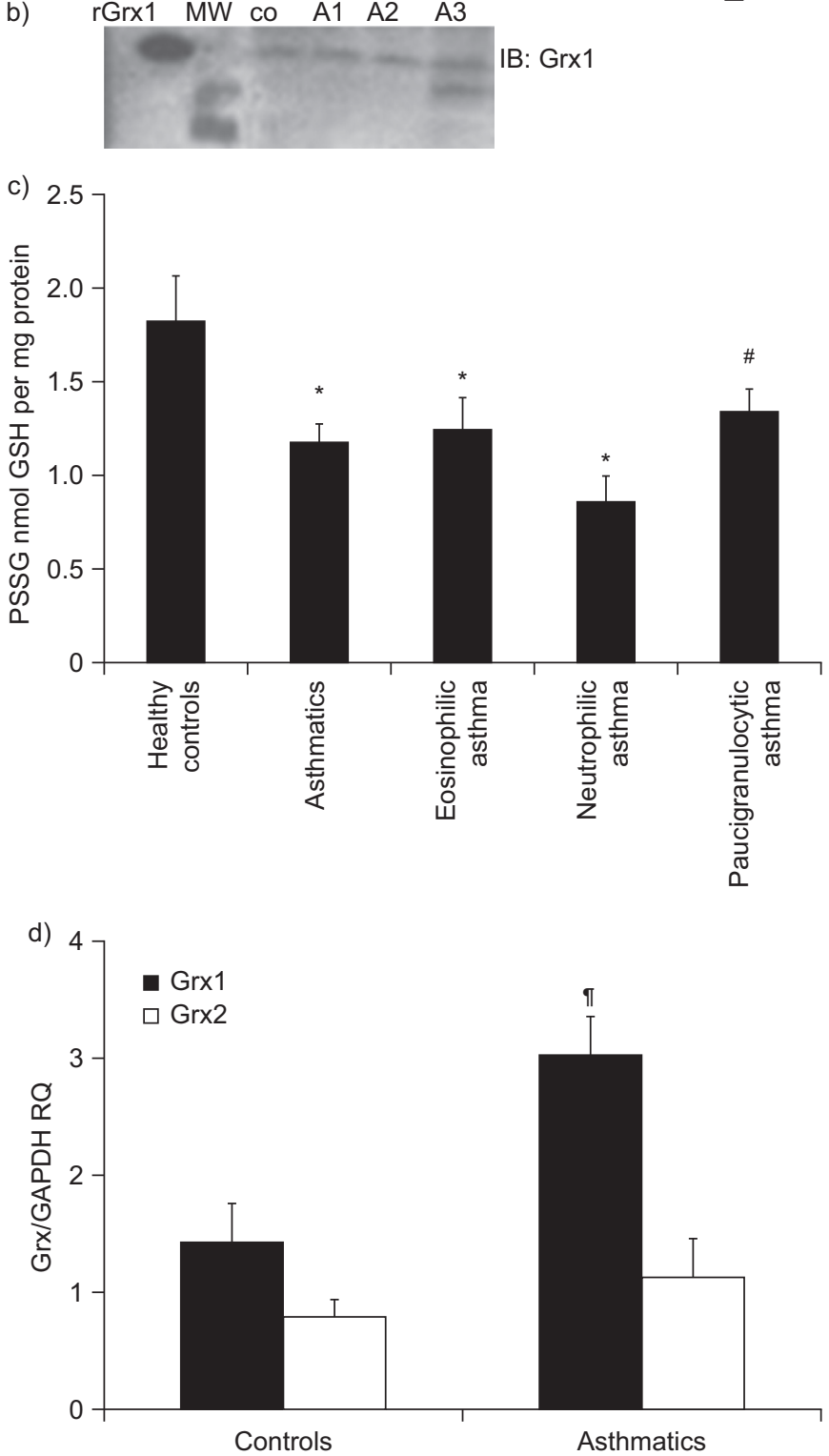

FIGURE 1. The glutaredoxin (Grx)1/S-glutathionylation (PSSG) axis in induced sputum and primary bronchial epithelial cells of asthmatics. a) Grx1 protein levels in induced sputum of healthy controls and asthma patients, assessed by Western blot. Data are expressed in arbitrary units (AU), where Grx1 levels were corrected for a sample of recombinant human Grx1 (rGrx1) on each gel, and presented as mean $\pm S D$. b) A representative Western blot loaded with a rGrx1 sample, a molecular weight marker (MW), a healthy control sputum sample (co) and three asthma sputa (A1-3). c) Level of PSSG in the induced sputum of healthy controls and asthma patients. Data are expressed as nanomoles of reduced glutathione (GSH) that was released per milligram of protein and presented as mean \pm SD. d) Grx1 and Grx2 mRNA expression corrected for glutaraldehyde phosphate dehydrogenase (GAPDH) in primary bronchial epithelial cells of three healthy controls and three patients with asthma. Data are expressed as relative quantity $(\mathrm{RQ})$ and presented as mean \pm SEM. *: $\mathrm{p}<0.05$ compared with healthy controls analysed by Kruskal-Wallis test followed by the Mann-Whitney U-test; *: $p<0.05$ compared with neutrophilic asthma analysed by Kruskal-Wallis test followed by the Mann-Whitney U-test; ${ }^{\circ}$ : $p<0.05$ compared with healthy controls by unpaired t-test.

Grx/PSSG alterations as a cause or a consequence of the disease are related to clinical manifestations.

Ine Kuipers*, Renaud Louis", Maité Manise",

Mieke A. Dentener*, Charles G. Irvin",

Yvonne M.W. Janssen-Heininger ${ }^{+}$, Christopher E. Brightling ${ }^{\S}$, Emiel F.M. Wouters* and Niki L. Reynaert*

*Dept of Respiratory Medicine, NUTRIM School for Nutrition, Toxicology and Metabolism, Maastricht University Medical Centre+, Maastricht, The Netherlands, "Dept of Pulmonary Medicine, CHU Sart-Tilman, I GIGA research group, University of Liege, Liege, Belgium, "Dept of Medicine, University of Vermont, ${ }^{+}$Dept of Pathology, University of Vermont, Burlington, VT, USA, and ${ }^{\S}$ Institute for Lung Health, University of Leicester, Leicester, UK.

Correspondence: N.L. Reynaert, Maastricht University Medical Centre+, Dept of Respiratory Medicine, PO Box 616, 6200 AZ Maastricht, The Netherlands. E-mail: n.reynaert@ maastrichtuniversity.nl

Support Statement: This study was supported by NUTRIM, a VENI grant from NWO/ZonMW (016.086.090) awarded to N.L. Reynaert and IUAP P6/35 supported by the Belgian Federal Government.

Statement of Interest: Statements of interest for Y.M.W. Janssen-Heininger and C.G. Irvin can be found at www.erj. ersjournals.com/site/misc/statements.xhtml

\section{REFERENCES}

1 Reynaert NL. Glutathione biochemistry in asthma. Biochim Biophys Acta 2011; 1810: 1045-1051.

2 Xiong Y, Uys JD, Tew KD, et al. S-glutathionylation: from molecular mechanisms to health outcomes. Antioxid Redox Signal 2011; 15: 233-270.

3 Peltoniemi MJ, Rytila PH, Harju TH, et al. Modulation of glutaredoxin in the lung and sputum of cigarette smokers and chronic obstructive pulmonary disease. Respir Res 2006; 7: 133. 
4 Reynaert NL, Wouters EF, Janssen-Heininger YM. Modulation of glutaredoxin-1 expression in a mouse model of allergic airway disease. Am J Respir Cell Mol Biol 2007; 36: 147-151.

5 Brightling CE. Sputum induction in asthma: a research technique or a clinical tool? Chest 2006; 129: 503-504.

6 Nagai K, Betsuyaku T, Konno S, et al. Diversity of protein carbonylation in allergic airway inflammation. Free Radic Res 2008; 42: 921-929.

7 Peltoniemi M, Kaarteenaho-Wiik R, Saily M, et al. Expression of glutaredoxin is highly cell specific in human lung and is decreased by transforming growth factor- $\beta$ in vitro and in interstitial lung diseases in vivo. Hum Pathol 2004; 35: 1000-1007.

8 Lundberg M, Fernandes AP, Kumar S, et al. Cellular and plasma levels of human glutaredoxin 1 and 2 detected by sensitive ELISA systems. Biochem Biophys Res Commun 2004; 319: 801-809.

9 Aesif SW, Anathy V, Guala AS, et al. Ablation of glutaredoxin-1 attenuates lipopolysaccharide-induced lung inflammation and alveolar macrophage activation. Am J Respir Cell Mol Biol 2011; 44: 491-499.

\section{A 61-year-old female with chronic cough and purulent sputum}

\section{To the Editor:}

A 61-yr-old Caucasian female was referred to our outpatient clinic for persistent cough, right scapular pain and purulent sputum for the past 9 months.

Her past medical history was consistent with a diagnosis of pneumonia almost 20 yrs previously. She reported no allergies and was not taking any medications at home. She lived in an urban setting working as a hairdresser, and had no pets at home. She had received all the standard vaccinations based on the Italian law, including diphtheria, smallpox, tetanus, hepatitis A and typhus. She was married and had two daughters in good health. She went on vacation to Cuba 2 yrs previously, India (Rajasthan) 1 yr previously and Portugal 6 months ago.

Her history of present illness was consistent with cough and mucopurulent sputum without fever for 9 months prior to visiting our clinic. She reported a scapolar right pain that had occurred 15 days prior to admission to the hospital which was not related to positioning or breathing. A negative chest radiograph had been performed 3 months prior to the visit, and at that point she was treated with oral prednisone $(25 \mathrm{mg}$ every $24 \mathrm{~h}$ for 15 days) with no evidence of clinical improvement. Finally, she underwent a chest computed tomography (CT) scan with the evidence of lung opacities with enhancement in the medium lobe and in the lingual (fig. 1a).

At the time she was referred to our outpatient clinic (Respiratory Clinics, Respiratory Department, San Gerardo Hospital, Monza, Italy) she was complaining of respiratory symptoms including cough and purulent sputum, while the physical examination was completely unremarkable. A chest examination was negative and she had did not have skin or pharyngeal lesions. A tuberculin test was performed and the result was negative. Blood examinations were obtained that showed a white blood cell count of 5.510 cell $\cdot \mu \mathrm{L}^{-1}$ and haemoglobin of $13 \mathrm{~g} \cdot \mathrm{dL}^{-1}$, with no evidence of anti-HIV antibodies.

She underwent bronchoscopy which showed mucupurulent secretions from all the lobar bronchi. The bronchoalveolar lavage (BAL) fluid showed 1,280,000 cells in a 25-mL sample, with $29 \%$ macrophages (without emosiderinic pigment), 68\% neutrophils, $1 \%$ eosinophils, $2 \%$ of lymphocytes and $<1 \%$ of hystiocytes

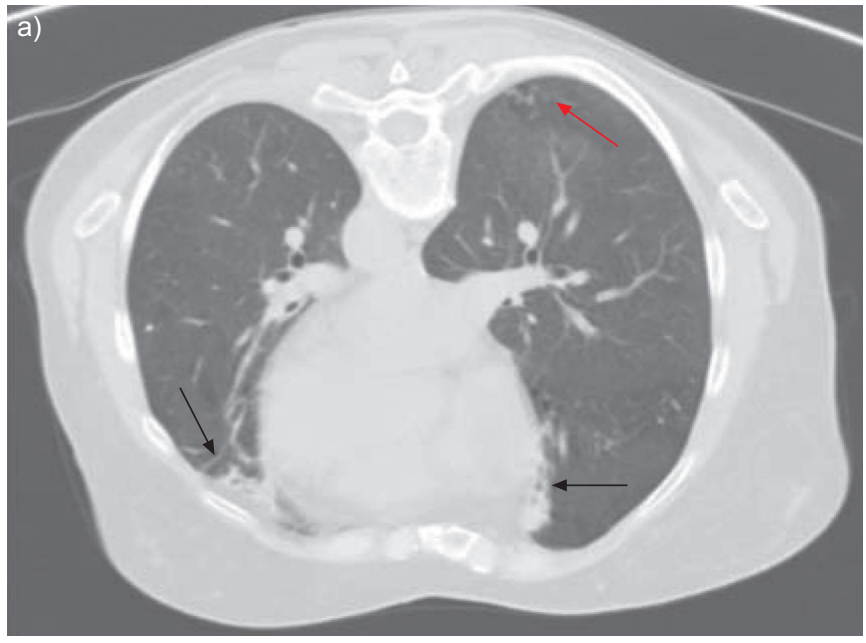

b)

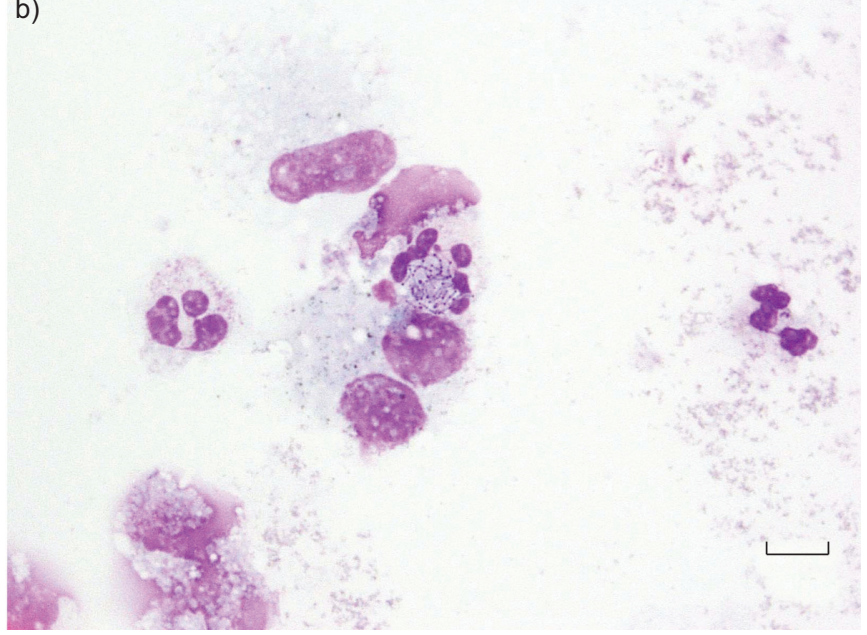

FIGURE 1. a) Chest computed tomography scan showing lung opacities in the medium lobe and in the lingula with bronchiectasis (black arrows). A "tree-in-bud" pattern is also present in the depending areas (red arrow). b) Bronchoalveaolar lavage fluid after May-Grünwald Giemsa staining. Several rod-shaped bacteria with irregular staining and "Chinese letters" arrangement of Corynebacterium spp. are visible inside a neutrophil. Scale bar $=500 \mu \mathrm{m}$. 